\title{
Long-term storage effects in steroid metabolite extracts from baboon (Papio sp.) faeces - a comparison of three commonly applied storage methods
}

\author{
Urs Kalbitzer $^{1 *}$ and Michael Heistermann ${ }^{2}$ \\ ${ }^{1}$ Cognitive Ethology Laboratory, German Primate Center (DPZ), Kellnerweg 4, 37077 Göttingen, Germany; and \\ ${ }^{2}$ Endocrinology Laboratory, German Primate Center (DPZ), Göttingen, Germany
}

\begin{abstract}
Summary
1. The measurement of steroid hormone metabolites from faeces in wild animal populations is a powerful, noninvasive tool in behavioural endocrinology of all major vertebrate taxa. However, because such research is often done in remote areas with limited infrastructure, storage of samples for hormone analysis over long periods at high temperature is a critical issue in field endocrinology. Previous studies have indicated that storage of alcoholic faecal extracts is more reliable than storage of unprocessed faeces if no freezer is available, but a standard method has not been established yet.

2. We tested the validity of three commonly applied storage conditions - liquid extracts, dried extracts and extracts placed on solid-phase extraction (SPE) cartridges - to preserve concentrations of glucocorticoid and androgen metabolites from faecal extracts of olive baboons (Papio anubis) at high temperature over 1 year.

3. Temporal variation in concentrations was detected for all metabolites and all storage conditions, including values measured from the control condition, that is, extracts stored at $-20^{\circ} \mathrm{C}$. This suggested that most variation was due to interassay variability, corroborated by comparisons of variation in 'quality controls' and samples.

4. Compared to frozen control samples, liquid extracts were stable for up to 24 weeks, extracts on SPE cartridges were stable for up to 50 weeks, while steroid metabolite concentrations in dried extracts decreased slightly over time.

5. If steroid samples have to be stored at ambient temperature, we suggest storage of liquid extracts for up to 24 weeks in a dark and cool place. For longer periods, SPE cartridges should be applied as evaporation, a potential confound arising with long-term storage of liquid extracts at higher temperatures, is not a problem in this storage condition. Storage of dried extracts is more cost-effective, but may result in small time-dependent changes in steroid concentrations.
\end{abstract}

Key-words: enzyme immunoassay, hormones, field endocrinology, glucocorticoid, androgen, testosterone, cortisol, primate

\section{Introduction}

The measurement of hormones in wildlife (i.e. field endocrinology) has become a widely applied method in many areas of zoological research including animal conservation (e.g. Millspaugh \& Washburn 2004; Walker, Boersma \& Wingfield 2005), reproductive biology (e.g. Strier \& Ziegler 1997; Ganswindt et al. 2005) and behavioural biology (e.g. Muller \& Wrangham 2004; Higham et al. 2009). The main class of hormones examined are the steroids, which play a major role in regulating many bodily functions (e.g. metabolism, immune function and salt and water balance) and underlie the majority of reproductive and behavioural processes (Nelson 2005). As such, the last decade has seen an increasing number of field studies examining the role of various steroids, including

*Correspondence author. E-mail: ukalbitzer@dpz.eu oestrogens, androgens and glucocorticoids, in mediating behavioural as well as life-history variation in species of all major vertebrate taxa.

The endocrine methods used in this relatively new field of research mainly rely on noninvasive sampling, that is, urine and faeces, which, in contrast to blood and saliva, are relatively easy to collect from animals living in their natural habitats. These sources contain high concentrations of metabolites derived from the biologically active forms of circulating hormone(s) (Brownie 1992; Whitten, Brockman \& Stavisky 1998; Moestl, Rettenbacher \& Palme 2005), allowing for reliable inferences about the endocrine state of an animal. Because capturing and anesthetization of the animal is not required, frequent collection of samples over long periods of time is feasible and usually does not interfere with the natural behaviour of the study animal. It also demands only one person, a few minutes effort per sample and is not cost-intensive. Faecal samples have become the most common source for physiological 
information due to the ease of collection from animals living under many different natural conditions. Additionally, faecal steroid metabolite concentrations represent pooled values for periods of several hours up to a few days (Schwarzenberger et al. 1996), which can be advantageous in many field studies where chronic rather than acute variation is of interest.

Many studies in field endocrinology are conducted with wild populations of mammals which are often found in remote areas where only simple laboratory equipment is available. Especially in research on tropical animals, field stations are most often based in remote areas of 'source countries' in Africa, South America or Asia, while samples are commonly analysed in laboratories located in the USA or Europe. Therefore, samples usually have to be stored for extended periods of time (weeks or even months) at field stations before being transported to the hormone laboratory for analysis. Thus, one of the most critical issues in such situations is to find optimal sample storage conditions compatible with high ambient temperatures, restricted power supply and simple laboratory equipment. Despite decades of field endocrinology, there is, however, not yet a standard method of preserving faecal samples in the field, due to various problems that accompany most applied methodologies (reviewed in Ziegler \& Wittwer 2005).

Traditionally, faecal samples have been stored in alcohol, frozen or dried (Ziegler \& Wittwer 2005; Hodges \& Heistermann 2011) until transport to the laboratory for steroid extraction. However, while methodologically simple, longterm storage of faeces at ambient temperature, unpreserved or in alcohol, can create substantial artificial variation in steroid hormone levels, most likely due to metabolite degradation (Khan et al. 2002; Hunt \& Wasser 2003; Pettitt, Wheaton \& Waterman 2007). In addition, methods of drying samples have produced inconsistent results. For example, in Cape ground squirrels (Xerus inauris), oven-dried samples stored at ambient temperatures were stable for up to 1 year (Pettitt, Wheaton \& Waterman 2007), while oven- and silica-dried samples from grizzly bears (Ursus arctos horribilis) and African elephants (Loxodonta africana) showed marked changes in metabolite concentrations after only 14 days (Hunt \& Wasser 2003). Lyophilizing (or freeze drying) faeces is considered highly reliable for long-term preservation of faecal hormones (Hunt \& Wasser 2003), but is not feasible at field stations due to a lack of equipment suitable for use in the field. Likewise, while keeping samples frozen until arrival at the laboratory has been shown to be an ideal preservation method (Khan et al. 2002; Hunt \& Wasser 2003), freezers are often not available at field stations due to a lack of constant power supply. In addition to potential storage problems, the transport of frozen faeces demands export and import permits as they are considered potentially pathogenic.

To avoid these potential problems, extraction of faecal steroid metabolites immediately after collection ('field extraction') has become increasingly common during the last years (Beehner \& Whitten 2004; Ziegler \& Wittwer 2005; Santymire \& Armstrong 2009; Freeman et al. 2010), as it is assumed that, once extracted, metabolite levels remain stable even at ambient temperature (Ziegler \& Wittwer 2005).
Furthermore, the process separates the metabolites from the potentially pathogenic faecal matrix, which can then be left on-site, making import and export permits easier to obtain or even unnecessary. Field extraction is also attractive because it requires little equipment (Ziegler \& Wittwer 2005), as it only involves mixing faecal material with alcohol, shaking the mixture by hand (or using a battery-powered homogenizer) to dissolve the hormones in the alcohol and then filtering (Beehner \& Whitten 2004) or centrifuging (e.g. by using a manually operating centrifuge; Shutt, Setchell \& Heistermann 2011) to separate the extract from the faecal matrix. The extract can then be stored as liquid, dried or on 'solid-phase extraction' (SPE) cartridges for long-term storage (Beehner \& Whitten 2004; Ziegler \& Wittwer 2005; Santymire \& Armstrong 2009).

However, few studies so far have tested the long-term stability of steroid metabolite concentrations in alcoholic extracts stored under various conditions and at higher temperatures. Cortisol and testosterone values from dried faecal extracts obtained from African wild dog (Lycaon pictus), for example, appear to be stable for up to 180 days at room temperature (Santymire \& Armstrong 2009), but levels were up to $80 \%$ lower when extracts were stored on SPE cartridges for only 30 days. For African elephants, progestagen values from liquid extracts were lower after 6,9 and 12 months compared to values measured immediately and after 3 months (Freeman et al. 2010), although this study had one important limitation, namely that faecal matter remained in the alcohol after mixing and the extract was only separated shortly before each measurement.

Among primates, a recent study by Shutt, Setchell \& Heistermann (2011) demonstrated that glucocorticoid concentrations in faecal extracts obtained from lowland gorillas (Gorilla gorilla gorilla) were stable for up to 1 year when stored as liquid or dried in polypropylene tubes at $22^{\circ} \mathrm{C}$. Faecal extracts from baboons (Papio sp.) stored on SPE cartridges were tested by Beehner \& Whitten (2004), but only for up to 40 days. The latter study demonstrated that oestradiol, progesterone and testosterone metabolite concentrations were stable over the entire 40-day study period, while glucocorticoid values decreased slightly. Similarly, a study in gelada baboons (Theropithecus gelada) showed that faecal extracts stored on SPE cartridges were stable for 3 weeks with respect to testosterone and glucocorticoid metabolite concentrations, followed by an increase in both metabolites after 4 weeks (Pappano, Roberts \& Beehner 2010).

Although these previous studies provided valuable information on the suitability of the different potential methods for short- and long-term storage of faecal extracts for endocrine analysis, none has tested the reliability of all common storage methods in one experiment over a long period of time and at temperatures more similar to those found at tropical field sites. It is important to assess the relative merits of the potential storage methods for preserving faecal steroid hormones long term, particularly since data collected so far are difficult to compare directly due to differences in the methodologies applied. Moreover, since previous studies were limited to only a few species, 
the extent to which these methods can be applied across species is unclear.

Here, we test the effect of storing faecal metabolites extracted with alcohol (i) as liquid, (ii) dried and (iii) on SPE cartridges at elevated temperature $\left(30^{\circ} \mathrm{C}\right)$ for an extended period of time relative to storage at $-20^{\circ} \mathrm{C}$. Specifically, we extracted androgen and glucocorticoid metabolites from olive baboon faeces. For extraction, we applied a field-friendly method and regularly measured metabolite concentrations after 3, 6, 9, 12, 24 and 50 weeks.

\section{Materials and methods}

\section{SAMPLES AND EXTRACTION}

We obtained faecal samples from six adult male (as we were mainly interested in testing androgens and glucocorticoids) olive baboons (Papio anubis) from the 'Station de Primatologie', Rousset sur arc, France. From each male, samples were collected in October 2010 on two consecutive days, immediately frozen and sent to the German Primate Center on dry ice. We processed eight different samples (A-H) including faeces from all six individuals.

To extract metabolites from faecal samples, we used a method that can be easily performed in a field laboratory. Specifically, we first homogenized the sample and placed $\sim 0.8 \mathrm{~g}$ of faeces into a 15 -mL polypropylene tube, to which $8 \mathrm{~mL}$ methanol $(80 \%)$ was added. We then shook the tubes by hand for two minutes, centrifuged them for $5 \mathrm{~min}$ at $\sim 1660 \times \mathrm{g}$ and subsequently aliquoted the supernatant (faecal extract) for the storage experiment. We used methanol for extraction because we stored extracts on SPE cartridges in one condition; these cartridges are usually primed with methanol, and accordingly, methanol has been used for steroid extraction in previous studies (Beehner \& Whitten 2004; Pappano, Roberts \& Beehner 2010).

\section{STORAGE CONDITIONS AND DURATIONS}

We divided each extract into four aliquots, one for each storage condition. We measured extracts immediately after extraction (time 0 control) and then repeatedly after 3, 6, 9, 12, 24 and 50 weeks of storage to assess any potential storage-type and time-dependent effect on steroid metabolite levels (Fig. 1). For the 'frozen' control condition, we pipetted $1 \mathrm{~mL}$ of each sample extract into $2-\mathrm{mL}$ polypropylene tubes (SafeSeal microtubes, Sarstedt, Germany) and stored them in a freezer

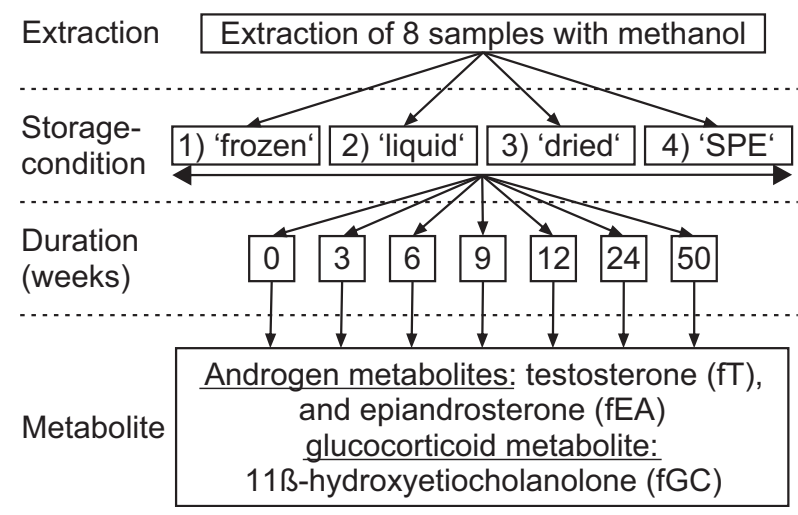

Fig. 1. Flow chart of experimental procedures. See text for details. at $-20^{\circ} \mathrm{C}$. For the 'liquid' condition, we placed extracts into $2-\mathrm{mL}$ polypropylene tubes and stored them in a laboratory oven at $30^{\circ} \mathrm{C}$. In both cases, parafilm was wrapped around tubes to minimize risk of evaporation and tubes were stored dark. For the 'dried' condition, we prepared aliquots of $0.25 \mathrm{~mL}$ from each sample in 2-mL polypropylene tubes, evaporated the alcohol at $30^{\circ} \mathrm{C}$ and subsequently stored tubes at $30^{\circ} \mathrm{C}$ in an oven. Finally, for the 'SPE' condition, we loaded $0.5 \mathrm{~mL}$ of each sample onto Sep-Pak Plus C18 cartridges (WAT020515, Waters, Milford, MA, USA) following procedures described elsewhere (Stavisky et al. 1995; Beehner \& Whitten 2004). Briefly, we diluted $0.5 \mathrm{~mL}$ of each extract with $1 \mathrm{~mL}$ distilled water $\left(\mathrm{dH}_{2} \mathrm{O}\right)$ and pushed this mixture slowly $\left(2-4 \mathrm{~mL} \mathrm{~min}^{-1}\right)$ through a cartridge primed with methanol and water using a syringe, according to the manufacturer's protocol. We rinsed the cartridges with $2 \mathrm{~mL} 0 \cdot 1 \%$ sodium azide solution in water acting as a preservative. Finally, we removed the remaining liquid from the cartridges by pushing $2 \mathrm{~mL}$ of air through them using a syringe. For storage, cartridges were put into small, airtight plastic bags (Whirl-Pak, Nasco, Elba, AL, USA) along with two teaspoons of silica beads to remove remaining moisture from the cartridges. We exchanged the silica repeatedly during the first 5 days to facilitate the drying process until the colour of the beads no longer changed, which indicated that all cartridges were completely dry. By this, we assured that all samples were stored under the same condition and thus minimized any risk of hormone metabolite degradation due to the presence of and variation in moisture content. Any contaminant (e.g. bacteria) potentially brought into the cartridges by this process is unlikely to have an effect on hormone stability as (i) bacterial enzymes potentially acting on the hormone metabolites are unlikely to be active in the absence of moisture and (ii) all cartridges contained sodium azide as a preservative against microbial activity.

We measured steroid metabolite concentrations immediately after extraction to serve as a time 0 control for the 'frozen', 'liquid' and the 'dried' conditions. For the 'SPE' condition, we used a separate time 0 control to account for any potential systematic loss in steroid recovery as a result of the procedure (i.e. lower recovery rate). Specifically, we loaded the cartridges with sample extract as described above, dried them for 5 days, eluted the steroids from the column (see below) and measured hormone concentrations thereafter.

\section{STEROID ASSAYS}

We assayed extracts for two androgen metabolites, immunoreactive testosterone (fT) and epiandrosterone (fEA), and one cortisol metabolite, 11ß-hydroxyetiocholanolone (fGC), using enzyme immunoassays (EIA) and running each sample in duplicate. All three steroid measurements have been previously validated for monitoring androgen and glucocorticoid output in various primate species (Moehle et al. 2002; Heistermann, Palme \& Ganswindt 2006). Details of the individual assay procedures are described elsewhere (fT and fEA: Palme \& Möstl 1994; Moehle et al. 2002; fGC: Ganswindt et al. 2003).

For hormone measurement from the 'frozen' and 'liquid' condition, one single aliquot per sample was repeatedly used at each experimental time point. To this end, prior to each hormone measurement, samples were removed from the freezer or laboratory oven and brought to room temperature by letting them stand on the bench for about $30 \mathrm{~min}$. Sample aliquots were brought back to the freezer or oven following measurements. For the 'dried' and 'SPE' conditions, we used necessarily several vials/cartridges per sample, one for each time point; we dissolved the dried extracts in $0.5 \mathrm{~mL} 80 \%$ methanol by sonication in a water bath for $5 \mathrm{~min}$ followed by $30 \mathrm{~s}$ of vortexing (Shutt, Setchell \& Heistermann 2011) and eluted hormones in the 'SPE' condition from the cartridges by rinsing the cartridges with $3 \mathrm{~mL}$ methanol $(100 \%$, flow 
rate: 2-4 $\mathrm{mL} \mathrm{min}^{-1}$ ). Prior to each assay, alcoholic extracts or SPE eluents were diluted with assay buffer (phosphate-buffered saline, $\mathrm{pH} 7 \cdot 2$ ) to bring hormone concentrations into the working range of the assay.

At each experimental time point, we measured all samples for each metabolite in one assay. Interassay coefficients of variation (CVs) for the fT assay were $7.4 \%$ and $19.9 \%$ for high and low value quality controls (QCs), respectively, resulting in a mean CV of $13.7 \%$. For fEA measurements, interassay $\mathrm{CVs}$ were $11.7 \%$ and $12.4 \%$ (mean $\mathrm{CV}$ $12 \cdot 1 \%$ ), and for the fGC assay, $12 \cdot 2 \%$ and $16 \cdot 0 \%$ (mean CV $14 \cdot 1 \%$ ).

\section{DATA ANALYSIS}

Steroid metabolite concentrations are expressed in mass steroid metabolites per mass faecal wet weight $\left(\mathrm{ng} \mathrm{g}^{-1}\right)$. To assess any overall storage-type and time-dependent effect on metabolite concentrations, we first performed a general linear mixed model for repeated measures (GLMM-RM) using storage condition, duration and the interaction condition*duration as fixed effects and, as we measured the same eight samples repeatedly at each time point and in all four storage conditions, the sample (A-H) as a random factor. For illustration of results, we calculated for each storage condition the change (in percentage) between time 0 control values and all following time points by $\left(\mathrm{M}_{\mathrm{tx}} * 100 / \mathrm{M}_{0 \mathrm{x}}\right)$ 100 , where $\mathrm{M}_{0 \mathrm{x}}$ is the concentration of metabolite $\mathrm{M}$ at time point 0 (control) for sample $\mathrm{x}$ and $\mathrm{M}_{\mathrm{tx}}$ the concentration at time point $\mathrm{t}$ for sample x. Furthermore, we calculated the difference (as a percentage) between samples from the 'frozen' control and test conditions at each time point by $\left(\mathrm{M}_{\mathrm{cx}} * 100 / \mathrm{M}_{\mathrm{fx}}\right)-100$, where $\mathrm{M}_{\mathrm{fx}}$ is the concentration for metabolite $\mathrm{M}$ for sample $\mathrm{x}$ from the 'frozen' condition and $\mathrm{M}_{\mathrm{cx}}$ the concentration for sample $\mathrm{x}$ from the test condition $\mathrm{c}$.

For each storage condition, we calculated the CVs for each of the eight samples across the seven respective metabolite measurements (weeks 0-50). As all samples per time point were measured together on one assay plate, these CVs should reflect the sum of interassay variation and potential variation due to storage effects. Therefore, we compared these storage condition $\mathrm{CVs}$ with the $\mathrm{CVs}$ of our quality controls to assess whether any observed temporal variation in sample values across the experiment can be explained by normal interassay variation.

As simple freezing stabilizes steroid levels over long periods of time (e.g. Hunt \& Wasser 2003; Shutt, Setchell \& Heistermann 2011), we conducted two-tailed paired $t$-tests to compare the 'frozen' control condition with the other conditions at each time point. In the 'SPE' condition, we used different time 0 controls to compensate for any potential differences in recovery rates (see above). The 'SPE' time 0 control concentrations were not significantly different from $-20^{\circ} \mathrm{C}$ time 0 control concentrations for fEA $(104.6 \% ; t(7)=-1.218, P=0.263)$ and fGC $(101.2 \% ; t(7)=-0.817, P=0.441)$; thus, we did not expect any systematic variation between the 'SPE' and other conditions at experimental time points due to differences in recovery rates. Accordingly, we used absolute concentrations for all comparisons between conditions. For fT, however, 'SPE' time 0 controls showed markedly higher concentrations compared to the $-20^{\circ} \mathrm{C}$ controls $(179.2 \%, P<0.01)$. This was most likely due to the disruptive effect of sodium azide (used as preservative in the SPE cartridges) on the activity of the horseradish peroxidase linked directly to our testosterone label. Therefore, we excluded the 'SPE' condition from all $\mathrm{fT}$ analyses.

Finally, to evaluate a potential time-dependent storage effect in the 'dried' condition, we calculated the spearman rank correlation coefficient using storage duration and differences in concentrations between 'frozen' and 'dried' samples as variables. All statistics were computed using IBM SPSS Statistics version 20 (IBM Corp., Armonk, NY, USA).

\section{Results}

Changes in hormone levels in relation to time 0 controls as a function of storage condition and duration are shown in Fig. 2 for all three steroid metabolites measured. Overall, our storage experiment revealed that levels of each metabolite showed condition- and time-dependent changes over the 12 months of storage, but the changes did not usually exceed $\pm 30 \%$ of the time 0 controls at any point in time. The magnitude and direction of changes in hormone concentrations were similar across conditions, but showed no consistent pattern in relation to conditions or duration of storage (Fig. 2).

GLMM analyses revealed a significant interaction between storage condition and duration for all three metabolites (Table 1); thus, variation between time points has to be considered per condition and vice versa. Our measures of variation within conditions (as calculated by condition CVs; Table 2) are similar to the mean CVs of quality controls used to assess assay variability over the 12-month experimental period, which means that temporal variation in all steroid metabolite concentrations does not significantly exceed magnitude of interassay variation. (a)

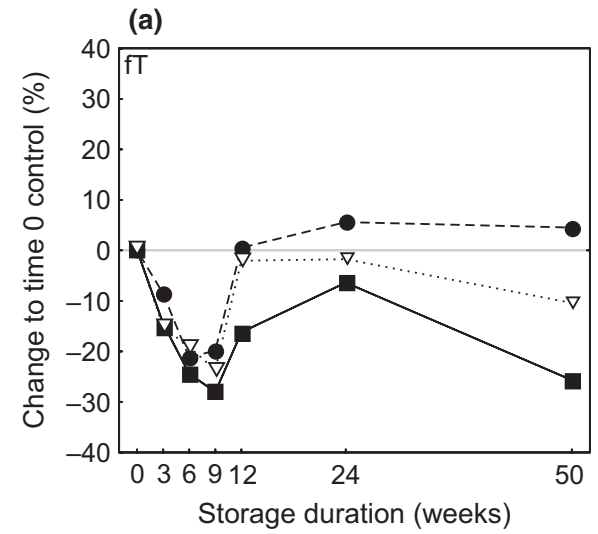

(b)

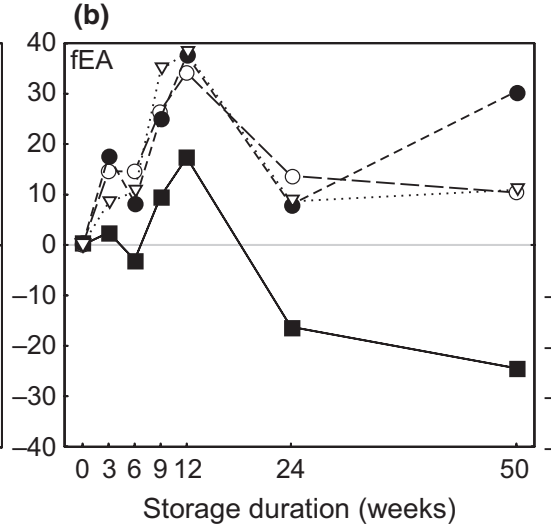

(c)

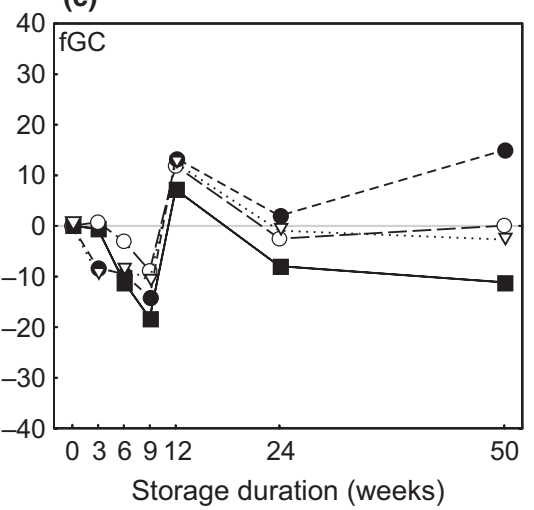

Fig. 2. Changes in hormone concentrations in baboon faecal extracts between time 0 control and experimental time points for (a) testosterone ( $f T$ ), ( $b$ )

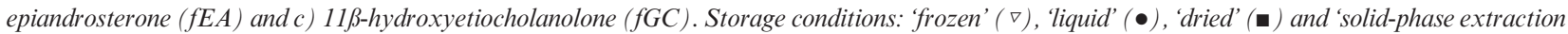
$(S P E)^{\prime}(0)$. 
Table 1. Results of GLMMs: effects of storage condition, duration and the interaction condition*duration on hormone concentrations in baboon faecal extracts for testosterone (fT), epiandrosterone (fEA) and 11ß-hydroxyetiocholanolone (fGC)

\begin{tabular}{llll}
\hline Metabolites & Storage condition & Storage duration & Condition*duration \\
\hline fT & $F(2,147)=31 \cdot 09, P<0.001$ & $F(6,147)=51 \cdot 09, P<0.001$ & $F(12,147)=5.50, P<0.001$ \\
fEA & $F(3,196)=40.34, P<0.001$ & $F(6,196)=20 \cdot 74, P<0.001$ & $F(18,196)=4 \cdot 29, P<0 \cdot 001$ \\
fGC & $F(3,196)=9.59, P<0.001$ & $F(6,196)=25 \cdot 08, P<0.001$ & $F(18,196)=3 \cdot 30, P<0.001$ \\
\hline
\end{tabular}

Table 2. Comparison of variation in measurements of baboon faecal extracts over the 12-month study with interassay variation: coefficients of variation $(\mathrm{CV})$ for all storage conditions and assay quality controls (QC) for testosterone (fT), epiandrosterone (fEA) and 11ß-hydroxyetiocholanolone (fGC)

\begin{tabular}{|c|c|c|c|}
\hline \multirow[b]{2}{*}{ Condition } & \multicolumn{3}{|c|}{$\mathrm{CV}[\%]$} \\
\hline & $\mathrm{fT}$ & fEA & fGC \\
\hline Frozen & 11.9 & $14 \cdot 2$ & $10 \cdot 5$ \\
\hline Liquid & $13 \cdot 2$ & $13 \cdot 8$ & $12 \cdot 9$ \\
\hline Dried & $14 \cdot 1$ & $16 \cdot 9$ & $11 \cdot 2$ \\
\hline $\begin{array}{c}\text { Solid-phase } \\
\text { extraction }\end{array}$ & $-^{*}$ & $13 \cdot 9$ & $11 \cdot 2$ \\
\hline QC mean & $13 \cdot 7$ & $12 \cdot 1$ & $14 \cdot 1$ \\
\hline
\end{tabular}

*Data not available (see Materials and methods).

In comparing test conditions to the 'frozen' control condition at each time point, concentrations in the 'liquid' condition are only significantly higher for fT after 50 weeks, for fEA after 3 and 50 weeks and for fGC after 50 weeks (Fig. 3a, Table 3). After 50 weeks, we observed that the volume of several extracts was decreased, probably due to evaporation. For the 'dried' condition, concentrations of all three metabolites were lower at almost all time points (Fig. 3b, Table 3), and in more than $50 \%$ of cases ( 10 out of 18 ), this decrease was statistically significant (Table 3 ). As we used the same time 0 controls for the 'dried' and the 'frozen' control condition, we are not able to test whether lower values in dried samples were due to a systematic loss of metabolites as a result of drying and resolving procedures (as tested for the 'SPE' condition). However, such an effect would be expected to result in constant deviations from control values, whereas the deviations observed here were correlated with storage duration (fT: $r_{\mathrm{s}}=-0.591, P<0.001 ;$ fEA: $r_{\mathrm{s}}=-0.645, P<0.01 ;$ fGC: $\left.r_{\mathrm{s}}=-0.364, P<0.01\right)$, suggesting a storage effect in this condition.

In the 'SPE' condition, fEA concentrations are only significantly different from the frozen controls after 3 and 24 weeks and fGC concentrations after 6 weeks (Fig. 3c, Table 3), suggesting no systematic variation from frozen controls. Comparisons in fT concentrations were not included here due to problems with sodium azide (see methods).

\section{Discussion}

The results of our study indicate that the storage of baboon faecal extracts outside a freezer for the measurement of steroid hormone (glucocorticoid and androgen) metabolites is possible for up to 1 year without introducing a major storage effect.
Specifically, we demonstrate that two out of three tested storage methods, that is, storing alcoholic faecal extract as liquid in a polypropylene tube or dried on SPE cartridges, are suitable for preserving hormone levels long term at high temperatures and that storage at $-20^{\circ} \mathrm{C}$ did not appear to be better. Our results thus partly confirm findings from a recent storage experiment of gorilla faecal extracts, which showed that, in terms of glucocorticoid measurements, extracts stored as liquid at room temperatures $\left(22^{\circ} \mathrm{C}\right)$ remained equally stable as extracts stored long term in a freezer (Shutt, Setchell \& Heistermann 2011). Our data extend these previous findings, however, in that we demonstrate that the applied storage methods are also suitable for long-term preservation of faecal androgens and that they also stabilize steroid levels under higher temperature conditions similar to those found in the field. Moreover, they suggest that storage of liquid extracts is reliably applicable across species, at least within catarrhine primates or, perhaps, primates more generally. Only storage of dried extracts appeared to be affected by higher temperature, as hormone concentrations were reported to be stable at room temperature in other studies (Santymire \& Armstrong 2009; Shutt, Setchell \& Heistermann 2011), while the results of our study (at elevated temperature) suggest a weak storage effect.

Despite an overall stability of metabolite levels over the experimental period, concentrations of each of the three metabolites measured showed variation over time. The percentage changes in hormone concentrations were usually $<30 \%$ and the pattern of change was generally not directed (i.e. hormone levels did not consistently increase or decrease as a function of storage duration for any of the three hormone metabolites measured), suggesting that most observed significant differences did not reflect true changes in hormone levels due to, for example, bacterial or chemically induced alterations in metabolite structure. Rather, since the variation in the repeated sample measurements across the 12-month experimental period was similar to our interassay variability and, moreover, the temporal pattern of the changes observed was the same across conditions (i.e. hormone values between months increased or decreased in the same way in each condition), we believe that most significant differences to time 0 controls within conditions are simply explained by assay variation (see also Shutt, Setchell \& Heistermann 2011).

This is clearly different from the situation observed for storage of unprocessed faeces in alcohol, which has often been reported to result in marked changes of hormone levels even after short durations of storage and which appears to be clearly affected by temperature, being more pronounced in samples stored at higher temperatures compared to frozen samples (Khan et al. 2002; Hunt \& Wasser 2003; Lynch et al. 2003). 

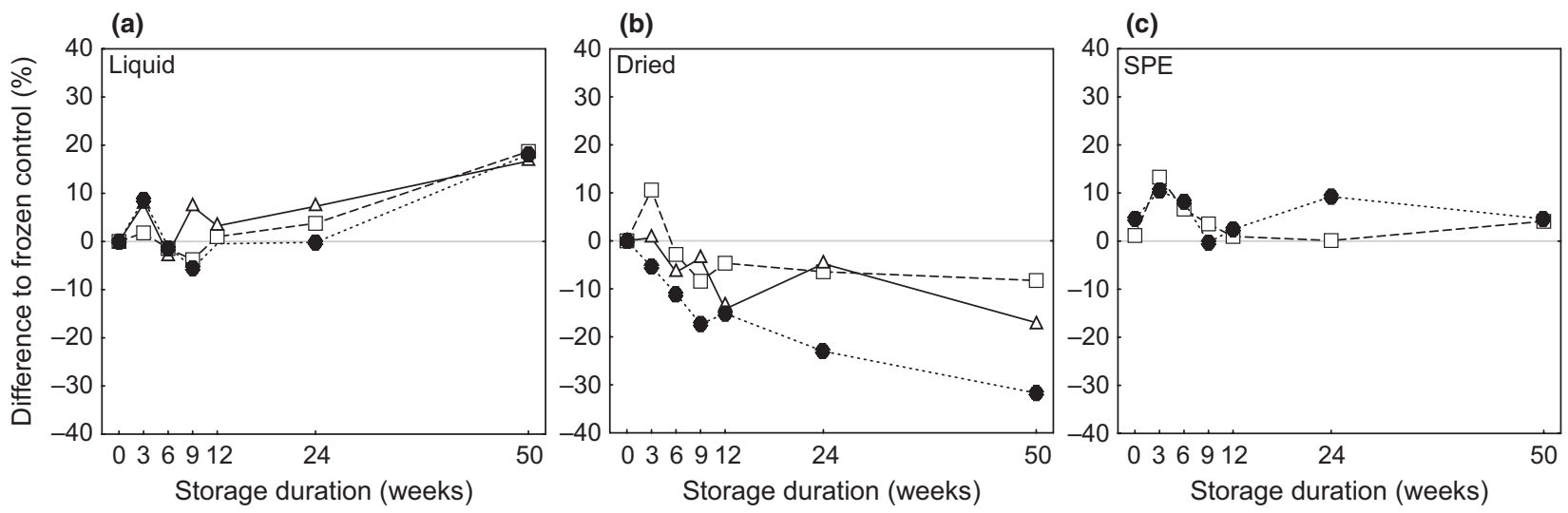

Fig. 3. Differences in hormone concentrations of baboon faecal extracts between the 'frozen' and test conditions for (a) 'liquid', (b) 'dried' and (c) 'solid-phase extraction (SPE)' conditions for fT $(\triangle)$, fEA $(*)$ and fGC ( $\square)$ levels.

Table 3. Differences in hormone concentrations in baboon faecal extracts between the 'frozen' and test conditions for testosterone (fT), epiandrosterone (fEA) and 11ß-hydroxyetiocholanolone (fGC)

\begin{tabular}{|c|c|c|c|c|c|c|c|c|}
\hline \multirow[b]{3}{*}{ Weeks } & \multicolumn{2}{|l|}{$\mathrm{fT}$} & \multicolumn{3}{|l|}{ fEA } & \multicolumn{3}{|l|}{ fGC } \\
\hline & \multicolumn{2}{|c|}{$\begin{array}{l}\text { Difference }(\%) \text { between } \\
\text { frozen and }\end{array}$} & \multicolumn{3}{|c|}{ Difference $(\%)$ between frozen and } & \multicolumn{3}{|c|}{ Difference $(\%)$ between frozen and } \\
\hline & Liquid & Dried & Liquid & Dried & SPE & Liquid & Dried & SPE \\
\hline 0 & - & - & - & - & 4.6 & & & $1 \cdot 2$ \\
\hline 3 & $7 \cdot 8$ & $0 \cdot 6$ & $8 \cdot 7^{*}$ & $-5 \cdot 4$ & $10 \cdot 5^{*}$ & $1 \cdot 8$ & $10 \cdot 6$ & $13 \cdot 3$ \\
\hline 6 & $-2 \cdot 6$ & $-6 \cdot 5^{* *}$ & $-1 \cdot 0$ & $-11 \cdot 0^{*}$ & $8 \cdot 2$ & $-1 \cdot 5$ & $-2 \cdot 8$ & $6.7^{*}$ \\
\hline 9 & $7 \cdot 3$ & $-3 \cdot 6$ & $-5 \cdot 5$ & $-17 \cdot 3^{*}$ & $-0 \cdot 3$ & $-3 \cdot 8$ & $-8 \cdot 4^{* *}$ & $3 \cdot 6$ \\
\hline 12 & $3 \cdot 2$ & $-14 \cdot 2^{* *}$ & $-0 \cdot 5$ & $-15 \cdot 1 * *$ & $2 \cdot 5$ & $1 \cdot 0$ & $-4 \cdot 6$ & $1 \cdot 0$ \\
\hline 24 & $7 \cdot 3$ & $-4 \cdot 8$ & $-0 \cdot 2$ & $-23 \cdot 0 * *$ & $9 \cdot 3^{*}$ & $3 \cdot 8$ & $-6 \cdot 4$ & $0 \cdot 1$ \\
\hline 50 & $16 \cdot 7 * *$ & $-17 \cdot 0^{* *}$ & $18 \cdot 1 * *$ & $-31.7 * *$ & $4 \cdot 6$ & $18.7 * *$ & $-8 \cdot 2^{*}$ & $4 \cdot 1$ \\
\hline
\end{tabular}

Significant differences determined from two-tailed paired $t$-tests are indicated by: ${ }^{* *} P<0.01$ and $* P<0.05$ (for a detailed list with exact $P$-values, see Supporting Information Table S1). SPE, Solid-phase extraction.

Our study thus corroborates that storing faecal extracts rather than unprocessed faeces in alcohol is highly recommended for long-term preservation when unprocessed faecal samples cannot be frozen immediately (Ziegler \& Wittwer 2005; Shutt, Setchell \& Heistermann 2011). Although in our study and others (e.g. Beehner \& Whitten 2004; Santymire \& Armstrong 2009; Shutt, Setchell \& Heistermann 2011), several conditions tested appeared to be suitable for preserving mammalian steroid hormones long term at ambient temperatures, their relative merits are difficult to assess due to interstudy methodological variation and because to date no study has tested more than two methods simultaneously. Here, we examined how the three most often used techniques for faecal sample extract preservation affected the concentrations of several steroid metabolites when stored long term at tropical temperatures and how they compare to the standard method, that is, storage at subzero temperatures. Since we measured all samples at each time point over the 12 months of storage in a single assay plate, we can exclude any potential interference resulting from interassay variability (see above), allowing us to compare conditions and the impact of storage duration on steroid metabolite levels in more detail.
By using the extracts stored frozen as a control, we found that hormone concentrations in liquid samples stored at high temperature $\left(30^{\circ} \mathrm{C}\right)$ were highly stable during the first 24 weeks for all three metabolites, with changes in levels varying no more than $\pm 10 \%$ from the controls. Concentrations thereafter (i.e. measured after 1 year of storage) were elevated by, on average, $17.8 \%$ for each hormone. The most likely explanation for this rise in levels is increased evaporation of the alcoholic solvent between 24 and 50 weeks of storage, resulting in more concentrated samples and consequently higher metabolite concentrations. This was confirmed by our observation that extract volume in some samples had visibly decreased. Evaporation of the alcohol has also been reported to be the cause for increasing hormone concentrations in faecal extracts of gorillas stored long term in glass tubes (Shutt, Setchell \& Heistermann 2011) and for the rise in steroid levels in ethanol-preserved bird plasma stored at elevated temperatures (Goymann et al. 2007). The fact that we used the same sample aliquots for repeated hormone measurements at experimental time points with the consequence that extracts were subject to repeated heat-cooling cycles had presumably contributed to an increased evaporation risk in our study. In addition, the fact 
that tubes were repeatedly opened may have resulted in worn tube lids, which might have facilitated effective evaporation of the alcohol, probably enhanced by a higher gas pressure in the samples stored at $30^{\circ} \mathrm{C}$ compared to samples stored frozen. There are several possibilities to minimize such evaporation risk. First, extracts should be stored in a cold environment whenever possible. As shown by Shutt, Setchell \& Heistermann (2011), liquid extracts stored at room temperature in polypropylene tubes of the same producer as used in our study were stable for up to 1 year with no evaporation having occurred. Accordingly, storage in gas refrigerators, which are often available in countries with less-developed infrastructure and thus limited power supply, could help to avoid evaporation at the field site. Alternatively, extracts may be moved to a refrigerator or freezer placed in the nearest town periodically (e.g. every 3 months). Furthermore, tubes should not be opened before steroid analysis is carried out in the laboratory in order to minimize the risk that tube lids would become worn and in turn permeable for the solvent. Finally, tubes of different types or brands should be tested, as quality might differ between producers (see Goymann et al. 2007; Shutt, Setchell \& Heistermann 2011). Nevertheless, despite potential evaporation in our liquid samples stored at elevated temperatures for 1 year, the rises in concentrations of all three hormones tested were still relatively small, that is, ranging between $5 \%$ and $30 \%$. Thus, we envisage that by applying procedures that minimize evaporation of the alcohol, hormone levels will stay unchanged even after very long periods of storage (see Shutt, Setchell \& Heistermann 2011).

Drying the extract for long-term storage might be a suitable alternative because it eliminates the potential problem of evaporation. However, in contrast to other studies (Santymire \& Armstrong 2009; Shutt, Setchell \& Heistermann 2011), this method appeared to be slightly less reliable in our study. Compared to the frozen controls, levels of all three steroid metabolites measured from dried extracts were lower (on average $6 \cdot 1-19.6 \%$ ) at almost all time points of the experimental period. Since the deviation from the control values was not constant over time, but was significantly correlated with storage duration, it is unlikely that this effect was solely due to an insufficient (i.e. $<100 \%$ ) recovery of the hormones from the tube wall during reconstitution of the dried extract. Alternative explanations may thus apply: (i) metabolites sticking to the tube wall increasingly degraded with storage duration, (ii) metabolites may 'bind' to the polypropylene and this binding became more cohesive with time, and thus an increasing amount of metabolites could not be redissolved into the alcohol, or (iii) a species difference exists, as this storage method was reported to stabilize steroid hormones long term in other species (wild dogs: Santymire \& Armstrong 2009; gorillas: Shutt, Setchell \& Heistermann 2011). Considering that our storage temperature was higher than in the two previous studies $\left(30^{\circ} \mathrm{C}\right.$ vs. room temperature), we believe that the storage effect more likely occurred due to degrading or sticking metabolites rather than as a result of species difference. If this is true, such a storage effect could be avoided by storing dried samples in a colder environment.
Concerning the storage of extracts on SPE cartridges, our data revealed highly stable steroid values for the entire study period of 1 year compared with the storage at $-20^{\circ} \mathrm{C}$. Previous studies where SPE cartridges have been tested in baboons and geladas found significant decreases or increases in GC and T metabolites when stored for up to 6 weeks at ambient temperatures (Beehner \& Whitten 2004; Pappano, Roberts \& Beehner 2010). However, the changes observed were relatively small as in our study (and may also be simply explained by assay variation), suggesting that storage on SPE cartridges represents a generally reliable method for storing steroid extracts over long periods of time at ambient temperatures (but see Santymire \& Armstrong 2009). Nevertheless, there are several drawbacks for the use of SPE cartridges. First, the procedure involves more methodological steps that could potentially create higher methodological variation. Additionally, its use is more costintensive and time-consuming compared to the other storage techniques and it requires the use of methanol, a solvent much more hazardous than ethanol. Finally, as our testosterone measurement demonstrates, caution should be taken when sodium azide is used as a preservative because it inhibits horseradish peroxidase activity and thus can disturb enzyme immunoassays that use horseradish peroxidase-labelled hormones as a tracer. Sodium azide is also highly toxic, making transport of samples and disposal of material in the field complicated. Potentially, this problem could be solved by using an alternative preservative, such as Proclin (Supelco, USA), which is often used at very low concentrations in commercial assay kits to prevent microbe growth and which does not interfere with EIAs.

Collectively, our study and those of others (Santymire \& Armstrong 2009; Shutt, Setchell \& Heistermann 2011) strongly indicate that storage of faecal extracts is a much better option than storage of unprocessed faeces in alcohol (Khan et al. 2002; Hunt \& Wasser 2003) or dry (Terio et al. 2002) for preserving steroid hormone concentrations long term if a freezer is not available. Simple storage of the alcoholic extract in polypropylene tubes is the easiest way to preserve hormone levels for at least 6 months at temperatures of up to $30^{\circ} \mathrm{C}$ (this study; Shutt, Setchell \& Heistermann 2011). When samples need to be stored for longer, moving the liquid samples to a cold environment or, alternatively, placing them onto SPE cartridges seems to be a suitable alternative (this study; Santymire \& Armstrong 2009; Shutt, Setchell \& Heistermann 2011), although this approach involves more methodological steps and may not provide reliable preservation in certain species (Santymire \& Armstrong 2009). Drying extract is also an alternative method (see Santymire \& Armstrong 2009; Shutt, Setchell \& Heistermann 2011), but more systematic testing of, for example, a potential temperature effect on steroid stability with this method needs to be conducted before making a final conclusion.

The number of studies testing faecal storage methods for preserving steroid hormones under field conditions is still very small and limits the ability to draw generalized conclusions about the cross-species applicability of the various methods described. However, from the data now available, we envisage 
that storage of faecal extracts as liquid in simple plastic tubes is the most promising technique for a reliable, long-term preservation of steroids (at least glucocorticoids and androgens) across species, and we hope that our study stimulates research to confirm this.

\section{Acknowledgements}

We thank Andrea Heistermann for expert help in hormone analysis and Romain Lacoste from the 'Station de Primatologie' for providing us with the baboon faecal samples. We also thank Brandon Wheeler, Julia Fischer, the associate editor and two anonymous reviewers for comments on previous versions of the manuscript and Kurt Hammerschmidt for statistical advice.

\section{Data accessibility}

Used data are available at: http://dx.doi.org/10.5061/dryad.q1s1v (Kalbitzer \& Heistermann 2013).

\section{References}

Beehner, J.C. \& Whitten, P.L. (2004) Modifications of a field method for fecal steroid analysis in baboons. Physiology \& Behavior, 82, 269-277.

Brownie, A.C. (1992). The metabolism of adrenal cortical steroids. The Adrenal Gland (ed V.H.T. James), pp. 209-224. Raven Press, New York.

Freeman, E.W., Abbondanza, F.N., Meyer, J.M., Schulte, B.A. \& Brown, J.L. (2010) A simplified method for monitoring progestagens in African elephants under field conditions. Methods in Ecology and Evolution, 1, 86-91.

Ganswindt, A., Palme, R., Heistermann, M., Borragan, S. \& Hodges, J. (2003) Non-invasive assessment of adrenocortical function in the male African elephant (Loxodonta africana) and its relation to musth. General and Comparative Endocrinology, 134, 156-166.

Ganswindt, A., Rasmussen, H.B., Heistermann, M. \& Hodges, J.K. (2005) The sexually active states of free-ranging male African elephants (Loxodonta africana): defining musth and non-musth using endocrinology, physical signals, and behavior. Hormones and Behavior, 47, 83-91.

Goymann, W., Schwabl, I., Trappschuh, M. \& Hau, M. (2007) Use of ethanol for preserving steroid and indoleamine hormones in bird plasma. General and Comparative Endocrinology, 150, 191-195.

Heistermann, M., Palme, R. \& Ganswindt, A. (2006) Comparison of different enzyme immunoassays for assessment of adrenocortical activity in primates based on fecal analysis. American Journal of Primatology, 68, 257-273.

Higham, J.P., Semple, S., MacLarnon, A., Heistermann, M. \& Ross, C. (2009) Female reproductive signaling, and male mating behavior, in the olive baboon Hormones and Behavior, 55, 60-67.

Hodges, J.K. \& Heistermann, M. (2011). Field endocrinology: monitoring hormonal changes in free-ranging primates. Field and Laboratory Methods in Primatology: A Practical Guide (eds J.M. Setchell \& D.J. Curtis), pp. 353-370. Cambridge University Press, Cambridge.

Hunt, K.E. \& Wasser, S.K. (2003) Effect of long-term preservation methods on fecal glucocorticoid concentrations of grizzly bear and African elephant. Physiological and Biochemical Zoology, 76, 918-928.

Kalbitzer, U. \& Heistermann, M. (2013). Data from: Long-term storage effects in steroid metabolite extracts from baboon (Papio $s p$.) faeces - a comparison of three commonly applied storage methods. Dryad Digital Repository. http:// dx.doi.org/10.5061/dryad.q1s1v

Khan, M.Z., Altmann, J., Isani, S.S. \& Yu, J. (2002) A matter of time: evaluating the storage of fecal samples for steroid analysis. General and Comparative Endocrinology, 128, 57-64.

Lynch, J.W., Khan, M.Z., Altmann, J., Njahira, M.N. \& Rubenstein, N. (2003) Concentrations of four fecal steroids in wild baboons: short-term storage conditions and consequences for data interpretation. General and Comparative Endocrinology, 132, 264-271.
Millspaugh, J.J. \& Washburn, B.E. (2004) Use of fecal glucocorticoid metabolite measures in conservation biology research: considerations for application and interpretation. General and Comparative Endocrinology, 138, 189-199.

Moehle, U., Heistermann, M., Palme, R. \& Hodges, J. (2002) Characterization of urinary and fecal metabolites of testosterone and their measurement for assessing gonadal endocrine function in male nonhuman primates. General and Comparative Endocrinology, 129, 135-145.

Moestl, E., Rettenbacher, S. \& Palme, R. (2005) Measurement of Corticosterone Metabolites in Birds' Droppings: an Analytical Approach. Annals of the New York Academy of Sciences, 1046, 17-34.

Muller, M.N. \& Wrangham, R.W. (2004) Dominance, aggression and testosterone in wild chimpanzees: a test of the 'challenge hypothesis'. Animal Behaviour, 67, 113-123.

Nelson, R.J. (2005) An Introduction to Behavioral Endocrinology. Sinauer Associates, Sunderland, Massachusetts.

Palme, R. \& Möstl, E. (1994). Biotin-streptavidin enzyme immunoassay for the determination of oestrogens and androgens in boar faeces. Advances of Steroid Analysis (ed S. Görög), pp. 111-117. Akadémiai Kiadó, Budapest.

Pappano, D.J., Roberts, E.K. \& Beehner, J.C. (2010) Testing extraction and storage parameters for a fecal hormone method. American Journal of Primatology, 72, 934-941.

Pettitt, B.A., Wheaton, C.J. \& Waterman, J.M. (2007) Effects of storage treatment on fecal steroid hormone concentrations of a rodent, the Cape ground squirrel (Xerus inauris). General and Comparative Endocrinology, 150, 1-11.

Santymire, R.M. \& Armstrong, D.M. (2009) Development of a field-friendly technique for fecal steroid extraction and storage using the African wild dog (Lycaon pictus). Zoo Biology, 29, 289-302.

Schwarzenberger, F., Möstl, E., Palme, R. \& Bamberg, E. (1996) Faecal steroid analysis for non-invasive monitoring of reproductive status in farm, wild and zoo animals. Animal Reproduction Science, 42, 515-526.

Shutt, K., Setchell, J.M. \& Heistermann, M. (2011) Non-invasive monitoring of physiological stress in the Western lowland gorilla (Gorilla gorilla gorilla): validation of a fecal glucocorticoid assay and methods for practical application in the field. General and Comparative Endocrinology, 179, 167-177.

Stavisky, R., Russell, E., Stallings, J., Smith, E.O., Worthman, C. \& Whitten, P.L. (1995) Fecal steroid analysis of ovarian cycles in free-ranging baboons. American Journal of Primatology, 36, 285-297.

Strier, K.B. \& Ziegler, T.E. (1997) Behavioral and endocrine characteristics of the reproductive cycle in wild muriqui monkeys, Brachyteles arachnoides. American Journal of Primatology, 42, 299-310.

Terio, K.A., Brown, J.L., Moreland, R. \& Munson, L. (2002) Comparison of different drying and storage methods on quantifiable concentrations of fecal steroids in the cheetah. Zoo Biology, 21, 215-222.

Walker, B.G., Boersma, P.D. \& Wingfield, J.C. (2005) Field Endocrinology and Conservation Biology. Integrative and Comparative Biology, 45, 12-18.

Whitten, P.L., Brockman, D.K. \& Stavisky, R.C. (1998) Recent advances in noninvasive techniques to monitor hormone-behavior interactions. American Journal of Physical Anthropology, 107, 1-23.

Ziegler, T.E. \& Wittwer, D.J. (2005) Fecal steroid research in the field and laboratory: improved methods for storage, transport, processing, and analysis. American Journal of Primatology, 67, 159-174.

Received 7 February 2013; accepted 18 March 2013

Handling Editor: Sean Rands

\section{Supporting Information}

Additional Supporting Information may be found in the online version of this article.

Table S1. Results of two-tailed paired $t$-tests testing differences in hormone concentrations in baboon faecal extracts between the 'frozen' and test conditions for testosterone (fT), epiandrosterone (fEA), and 11ß-hydroxyetiocholanolone (fGC). 